\title{
DESAFIO DE IDEIAS PARA O GOVERNO ABERTO: O CASO DA POLÍCIA MILITAR DE MINAS GERAIS - BRASIL
}

\author{
IDEAS CROWDSOURCING FOR THE OPEN GOVERNMENT: \\ THE CASE OF THE MILITARY POLICE OF MINAS GERAIS - BRAZIL \\ UN DESAFIO DE IDEAS PARA EL GOBIERNO ABIERTO: \\ EL CASO DE LA POLÍCIA MILITAR DE MINAS GERAIS - BRASIL
}

RESUMO

Busca-se identificar os resultados de uma plataforma de envio de ideias em termos de geração de inovações e do fortalecimento da colaboração, pilar do governo aberto. Plataformas de envio de ideias têm sido utilizadas por governos para a colaboração da sociedade na resolução de problemas públicos, entretanto a literatura carece de estudos sobre a absorção das ideias dos cidadãos para a produção de inovação e sobre as formas de comunicação bidirecional entre instituição pública e sociedade. 0 caso estudado é a aplicação da plataforma Prêmioldeia no $8^{\circ}$ Batalhão de Polícia Militar de Minas Gerais ( $8^{\circ} \mathrm{BPMMG}$ ). Concluiu-se que a plataforma contribuiu para a abertura da instituição para a colaboração dos cidadãos. 0 artigo complementa a literatura demonstrando a aplicação de inovação aberta com finalidade social, para a captação da percepção de um grupo sobre um problema e envolvimento dos cidadãos na solução de grandes problemas públicos.

PALAVRAS-CHAVE: Inovação aberta, colaboração, governo aberto, plataformas de ideias, crowdsourcing.

Teresa Cristina Monteiro Martins - teresacristina.ufla@gmail.com

Mestre em Administração Pública pela Universidade Federal de Lavras - Lavras - MG, Brasil

Paulo Henrique de Souza Bermejo - bermejo@dcc.ufla.br

Pós-doutorado em Inovação pela Bentley University - Massachusetts - EUA.

Artigo submetido em 24.02.2016 e aprovado 05.12.2016

DOI: http://dx.doi.org/10.12660/cgpc.v21n70.59470 


\section{ABSTRACT}

Aims to identify the results of a shipping platform ideas in terms of generation of innovations and strengthening of collaboration pillar of open government. ideas shipping platforms have been used by governments for the cooperation of society in solving public problems; however, the literature lacks studies on the absorption of ideas from citizens for producing innovation and the forms of two-way communication between public institutions and society. The case study is the application of Prêmioldeia platform on the 8th Military Police Battalion of Minas Gerais (8 ${ }^{\circ} \mathrm{BPMMG}$ ). It was concluded the platform contributed to the opening of the institution for the cooperation of citizens. Article complements the literature demonstrating the open innovation application with social purpose, to capture the perception of a group on a problem and citizen involvement in major public problem solving.

KEYWORDS: open innovation, collaboration, open government, platforms ideas, crowdsourcing.

\section{RESUMEN}

Busca identificar los resultados de unas ideas de la plataforma del envío en términos de generación de innovaciones y el fortalecimiento de la colaboración pilar de un gobierno abierto. Ideas envío plataformas han sido utilizados por los gobiernos para la cooperación de la sociedad en la solución de los problemas públicos; Sin embargo, la literatura carece de estudios sobre la absorción de las ideas de los ciudadanos para la producción de la innovación y las formas de comunicación de dos vías entre las instituciones públicas y la sociedad. El estudio de caso es la aplicación de la plataforma Prêmioldeia el 8 Batallón de la Policía Militar de Minas Gerais (8BPMMG). Se concluyó la plataforma contribuido a la apertura de la institución para la cooperación de los ciudadanos. Artículo complementa la bibliografía que demuestra la aplicación de la innovación abierta con finalidad social, para capturar la percepción de un grupo en un problema y la participación ciudadana en la resolución de problemas importantes pública.

PALABRAS CLAVE: innovación abierta, colaboración, gobierno abierto, plataformas ideas, crowdsourcing.

\section{INTRODUÇÃO}

Em um contexto em que a inovação aberta começa a ser estudada como estratégia para a abertura dos governos (Karakiza, 2015) e em que apenas $4 \%$ dos países do globo não possuem nenhuma estratégia para abrir seus governos (Organization for Economic Co-operation and Development [OECD], 2015), notou-se o quanto é relevante abordar o tema inovação aberta no setor público. Além da relevância do tema, o grande fator motivacional para sua escolha foi o fato de existirem diversas lacunas na literatura, que, quando preenchidas, colaborarão tanto para o desenvolvimento teórico do tema quanto para a implementação de mudanças práticas no setor público.

Consultas públicas on-line e desafios de ideias são nomenclaturas para mecanismos baseados em inovação aberta que vêm sen- do utilizados para a abertura e inovação de instituições públicas; colaborando com o que se denomina governo aberto, eGovernament, citizensourcing, eDemocracy, eParticipation, Wiki Government, Government 2.0 (Wijnhoven, Ehrenhard, \& Kuhn, 2015). Ao explorar o potencial democrático da inovação aberta, as instituições públicas podem fortalecer os três pilares de governo aberto: a transparência, a participação e a colaboração (Abu-Shanab, 2015; Al-Hujran, Al-Debei, Chatfield, \& Migdadi, 2015; Conradie \& Choenni, 2014).

Uma análise dos artigos publicados sobre governo aberto em diferentes países permite observar que países com grandes diferenças culturais, como os EUA (Lee \& Kwak, 2012; Wise, Miric, \& Gegenhuber, 2011), a Jordânia (Al-Hujran et al., 2015; Alawneh, Al-Refai, \& Batiha, 2013) e a China (Song \& Guan, 2015), ou mesmo com diferenças econômicas, como a Inglaterra (Cordella \& lannacci, 
2010; Panagiotopoulos, Al-Debei, Fitzgerald, \& Elliman, 2012), o Sri Lanka (Karunasena \& Deng, 2012) e a Coreia (P.-L. Sun, Ku, \& Shih, 2015), enfrentam problemas parecidos na abertura de seus governos. Os problemas estão relacionados à baixa participação cidadã nos canais de comunicação com - governo e à dificuldade de feedback do setor público para os cidadãos participantes, ou seja, principalmente associados ao terceiro pilar do governo aberto, que é a comunicação bidirecional entre governo e sociedade para alcance de objetivos comuns.

As lacunas teóricas estão relacionadas à escassez de pesquisas sobre os resultados concretos da utilização de metodologias de inovação aberta para a abertura de estruturas governamentais. Em seu artigo mais recente, o precursor do conceito inovação aberta, Henry Chesbrough, apontou que o estudo da capacidade de absorção das ideias pelas instituições organizadoras e da sua aplicação sem fins lucrativos é um tema novo e está despertando grande interesse da comunidade acadêmica (West, Salter, Vanhaverbeke, \& Chesbrough, 2014).

Tomou-se como objeto de estudo o desafio de ideias realizado pelo $8^{\circ}$ BPMMG, denominado "Estratégias e ações para reduzir os crimes contra o patrimônio". O 8 BPMMG é uma instituição militar tradicional, detentora do uso legal da força para a garantia do poder de polícia; possui estrutura hierárquica, processos formais rígidos e marcados fortemente pela cultura militar (Pereira, dos Santos, \& de Brito, 2010), o que tende a distanciá-la dos cidadãos atendidos por ela, razão pela qual a instituição foi escolhida como objeto de estudo.
Com vistas a colaborar com o arcabouço de pesquisas da área, neste artigo, apresenta-se um estudo empírico, visando a responder às questões de pesquisa: "Como o $8^{\circ}$ BPMMG, conhecido amplamente por sua estrutura formal e processos rígidos, fez uso de plataforma colaborativa de inovação aberta para fortalecer a colaboração, pilar do governo aberto?." E, ainda, "Quais os resultados da aplicação dos conhecimentos absorvidos nessas iniciativas?".

O objetivo deste artigo é identificar os resultados de uma plataforma de envio de ideias em termos de geração de inovações e fortalecimento da colaboração, pilar do governo aberto. Para isso, foi realizada uma pesquisa de natureza qualitativa do tipo estudo de caso. A pesquisa identificou uma nova prática de interação entre os agentes públicos e a sociedade, bem como a abertura de uma instituição pública marcada pela sua rigidez para receber ideias da sociedade, o que possibilitou legitimar e inspirar inovações em prol de melhorias na segurança pública. Por meio da pesquisa, também foram identificadas limitações similares a outras apontadas na literatura sobre o uso de inovação aberta para abertura de governos.

O artigo está estruturado da seguinte forma: (1) introdução; (2) fundamentação teórica; (3) metodologia; (4) resultados e discussão; e (5) conclusão.

\section{GOVERNO ABERTO: TRANSPARÊNCIA, PARTICIPAÇÃO E COLABORAÇÃO}

O termo governo aberto é frequentemente utilizado para descrever iniciativas de disponibilização de informações governamentais via internet, sendo confundido com o 
governo eletrônico (A. J. Meijer, Curtin, \& Hillebrandt, 2012). Ao passo que o governo eletrônico é associado à disponibilização de dados e serviços via internet, governo aberto é um termo mais abrangente, utilizado para descrever a utilização do potencial democrático das tecnologias para garantir a transparência, a participação e a colaboração entre governo e sociedade (Abu-Shanab, 2015; Al-Hujran et al., 2015; Conradie \& Choenni, 2014).

Governo aberto é aquele em que o poder público dialoga com a sociedade em um processo colaborativo de soluções para os problemas públicos. É um tema multidisciplinar que envolve ciência política, com as relações de poder; aspectos econômicos e de prioridade na agenda de políticas públicas; aspectos tecnológicos, que facilitarão a interação (A. J. Meijer et al., 2012).

Transparência, participação e colaboração são os três pilares que sustentam o governo aberto. A transparência é o primeiro pilar, pois o fornecimento de informações compreensíveis a todos os cidadãos tende a empoderar a sociedade e permitir que ela opine sobre as temáticas cujos dados foram disponibilizados (A. J. Meijer et al., 2012). A transparência tem por objetivo promover o controle e fomentar os cidadãos com informações sobre as ações do governo (Linders, 2012). Esse pilar é o mais básico e mais facilmente implantado pelos governos, e tem sido explorado na literatura quanto às estratégias para apresentação de dados governamentais importantes para os cidadãos (Hernández, Juan Fuente, Labra-Gayo, Pérez, \& Núñez-Valdez, 2015), e também quanto à qualidade dos dados apresentados (Abu-Shanab, 2015; Alawneh et al.,
2013; Osman et al., 2014), planejamento e tecnologias utilizadas para sua disponibilização (Cavalheiro \& Joia, 2014; H. Sun, Fang, \& Hsieh, 2014). Quanto à transparência, os dados geralmente são disponibilizados em portais desenvolvidos pelo governo.

Participação é entendida como o engajamento dos cidadãos na tomada de decisões do governo; seu objetivo é promover a efetividade do governo e a melhoria da qualidade das decisões tomadas (Linders, 2012). Enquanto a transparência é um fluxo unidirecional de informações dos governos para a sociedade, empoderando-a, a participação é um fluxo na direção contrária, no sentido de fornecer informações da sociedade para o governo de modo a agregar efetividade às ações governamentais (Linders, 2012). Para gerar participação, podem ser utilizadas consultas públicas e pesquisas, como questionários de respostas padronizadas.

A colaboração é o último pilar do governo aberto, é a cooperação entre os diferentes níveis de governo, entre este e os cidadãos e outras instituições (Veljković, BogdanovićDinić, \& Stoimenov, 2014). Enquanto o fluxo na participação é unidirecional e vai dos cidadãos para o governo, na colaboração, ele é bidirecional, e governo e cidadãos trabaIham juntos na coprodução de soluções. A colaboração pressupõe parceria e cooperação (Linders, 2012).

As pesquisas que abordam a implantação de tecnologias para o fortalecimento dos piares do governo aberto demonstram que a maioria das iniciativas governamentais foca principalmente a participação e transparência, e não a colaboração (Karakiza, 2015). Assim, a colaboração, detectada quando existe uma 
DESAFIO DE IDEIAS PARA O GOVERNO ABERTO: 0 CASO DA POLÍCIA MILITAR DE MINAS GERAIS - BRASIL

interação entre os cidadãos e agentes públicos, é o pilar mais abrangente do governo aberto, por demandar também transparência e participação. A transparência serve para fomentar os cidadãos com informações que os encorajem a participar com suas sugestões de melhorias no setor público. A colaboração também vai além da participação, que geralmente está limitada ao envio de mensagens que fomentem o governo com dados e não permite discussões que impactam as decisões das instituições públicas, o que está mais relacionado ao nível de colaboração (Karakiza, 2015).

No Brasil, um levantamento realizado por (Pinho, 2008) mostrou que, apesar de a tecnologia ser utilizada para o governo aberto por diversos estados brasileiros, os portais dessas instituições não eram efetivos nem mesmo quanto à transparência, e que a participação e colaboração, a despeito de toda a tecnologia disponível, estavam restritas a comunicações assíncronas por e-mail, formulários e pesquisas de opinião (Pinho, 2008).

Em pesquisa mais recente, Neves (2013) mostra a evolução de 15 anos de governo aberto no Brasil. Por meio dessa análise, nota-se o grande progresso em termos de transparência de dados, por meio do Portal da Transparência do Governo Federal e da pressão pelo desenvolvimento e do acesso cada vez mais frequente a portais locais de disponibilização de dados claros, coesos e de fácil acesso. Entretanto, os artigos que apresentam a evolução do governo aberto brasileiro têm enfoque na evolução em termos de transparência e demonstram que as iniciativas em termos de participação e colaboração on-line ainda são muito incipientes
(Agner, 2015; Cunha, 2010; da Rocha \& de Oliveira, 2015; Neves, 2013; Vaz, Ribeiro, \& Matheus, 2010).

Para estimular o governo aberto e principalmente o nível da colaboração, metodologias de inovação aberta têm sido utilizadas (Deng, Lin, Zhao, \& Wang, 2015; Karakiza, 2015; Sieber \& Johnson, 2015).

\section{INOVAÇÃO, INOVAÇÃO ABERTA E GO- VERNO ABERTO: FUNDAMENTOS CON- CEITUAIS}

O conceito de inovação foi associado inicialmente à economia, com destaque para a obra de Schumpeter, para quem a inovação é a aplicação comercial ou industrial de um novo produto, processo ou método de produção, podendo ser ainda considerados os novos mercados, fontes de suprimentos, além de práticas de organização em geral.

Essa definição mostra a estreita ligação entre a inovação e a capacidade das empresas em desenvolver seus processos para acompanhar o contexto capitalista. Todavia, a inovação vai além de um mecanismo econômico, a capacidade de inovar é característica intrínseca do ser humano, que a utiliza para atender às suas necessidades (Simms, 2006). Gonzalez, Llopis, and Gasco (2013) mostraram que, a partir dos anos 2000, a literatura de inovação começa a se voltar para o estudo do setor público, o que ocorre, primeiro, pela necessidade do setor público de buscar inovações de suas estruturas governamentais e garantir eficiência, e, segundo, pelo avanço das novas tecnologias de informação e comunicação, que seriam capazes de colaborar para essa inovação. 
No caso das instituições públicas brasileiras, a necessidade de inovação pode ser associada à maior cobrança popular por transparência, representada nas manifestações públicas (Instituto Brasileiro de Opinião Pública e Estatística [(Ibope, 2013, 2014), e à necessidade de atendimento a pressões internacionais para a instalação de governos mais abertos, com maior participação do povo na criação das políticas públicas (Borman \& Janssen, 2013; Neves, 2013).

Assim, apesar de a literatura teórica sobre inovação se concentrar mais especificamente na inovação no setor privado, existem vários insights derivados dessas teorias que são relevantes para moldar e explorar a inovação no setor público (Bloch \& Bugge, 2013).

Uma prática de inovação que tem emergido nas últimas duas décadas é a inovação aberta, definida por Chesbrough (2003) como estratégia para que uma organização compartilhe suas ideias e, ao mesmo tempo, absorva ideias externas a ela, acelerando o desenvolvimento de inovações.

As características do termo inovação aberta, conforme cunhado por Chesbrough (2003), podem ser identificadas no nível da administração pública quando as instituições do Estado se abrem para trocar conhecimentos com outras instituições ou com a sociedade. Essa abertura permite estabelecer um fluxo de saídas e entradas de conhecimento que aceleram a inovação dos processos e serviços oferecidos por essas instituições, bem como os torna mais eficazes, pois a visão dos usuários sobre esses serviços será considerada, e as experiências de sucesso de outras instituições podem inspirar a me-
Ihoria dos processos.

Assim como na inovação aberta os recursos para inovação podem surgir de atores externos à instituição inovadora, no governo aberto, espera-se que os cidadãos possam acompanhar e influenciar as decisões do governo por meio de acesso à informação e da abertura das arenas decisórias (A. Meijer \& Bekkers, 2015). O objetivo final do governo aberto vai muito além de fornecer informações aos cidadãos; é também incentivar a participação dos cidadãos e garantir que o conhecimento gerado nos canais de comunicação seja bem utilizado (Osman et al., 2014). Dessa maneira, espera-se o fortalecimento dos três pilares do governo aberto, que são a transparência, a participação e a colaboração (Abu-Shanab, 2015; Al-Hujran et al., 2015).

O destaque que tem sido atribuído ao tema abertura dos governos na literatura sobre inovação governamental advém da necessidade de estudar as diversas iniciativas de governo aberto que vêm se destacando pelo mundo para aumentar a transparência e participação cidadã. No Brasil, até o ano de 2013, as iniciativas de governo aberto tinham como objetivo principal a disponibilização de dados, o que vem mudando de 2014 aos dias atuais, em que se multiplica o uso de consultas públicas para geração de ideias para o desenvolvimento de políticas públicas (A. C. Z. Santos, André Luiz; Veroneze, Ricardo Braga; Botelho, Giancarla Aparecida; de Souza Bermejo, Paulo Henrique, 2015).

Entre as práticas de inovação aberta utilizadas pelos governos, está o crowdsourcing, que surgiu entre o fim dos anos 1990 e início dos anos 2000, impulsionado pelo avanço 
da internet e a utilização de grandes grupos de pessoas na realização de tarefas antes realizadas individualmente (Hopkins, 2011).

O crowdsourcing de ideias, também chamado de desafio de ideias, envolve o lançamento de uma questão-problema para um grande e diversificado grupo, visando captar ideias mais robustas do que aquelas já vinham sendo utilizadas para a solução do problema (Parvanta, Roth, \& Keller, 2013; Seltzer \& Mahmoudi, 2013). Inicialmente voltado para a aceleração da inovação, em grandes empresas privadas, atualmente, cresce o número de plataformas de crowdsourcing com finalidades sociais, como as apresentadas em Martins and Bermejo (2015).

Nas plataformas crowdsourcing utilizadas por instituições públicas, espera-se que os cidadãos possam acompanhar e influenciar as decisões do governo por meio de acesso à informação e da abertura das arenas decisórias (A. Meijer \& Bekkers, 2015). Dessa maneira, tais iniciativas colaboram com 0 fortalecimento dos três pilares do governo aberto (Abu-Shanab, 2015; Al-Hujran et al., 2015).

\section{METODOLOGIA}

O estudo que deu origem a este artigo pode ser classificado como de natureza descritiva, uma vez que relata as características de um objeto de estudo e demonstra seus resultados (Constant, 2009). Quanto aos meios utilizados, por se aprofundar na pesquisa de um objeto de estudo, classifica-se como estudo de caso, em que se procurou explicar um acontecimento ao longo do tempo e em determinado contexto (Yin, 2003).
O objeto empírico foi o desafio de ideias sobre "Estratégias e ações para reduzir os crimes contra o patrimônio", ocorrido na plataforma de software Prêmioldeia e direcionado aos cidadãos atendidos pela instituição proponente do desafio, o 8 BPMMG. Esse objeto de estudo foi escolhido pela viabilidade de acesso a informações e por representar uma inovação que permitiu a abertura de uma instituição militar de estrutura rígida e tradicional para o estabelecimento de um fluxo bidirecional de conhecimentos entre agentes públicos e cidadãos. O funcionamento detalhado desse aplicativo pode ser encontrado em Santos et al. (2015).

Os procedimentos utilizados para a coleta de dados foram os seguintes:

(A) Observação do objeto empírico, realizada durante todo o período de execução do desafio, de 23/9 a 20/11/2013, etapa em que foram observadas as funcionalidades da plataforma, o perfil e o comportamento dos atores participantes.

(B) Análise quantitativa das ações realizadas pelos participantes na plataforma, por exemplo, número de ideias enviadas por cada perfil de usuário, realizada no período de 21/11/2013 a 15/1/2014, utilizando planilhas eletrônicas.

(C) Análise do conteúdo das ideias e comentários inseridos pelos participantes, também realizada de 21/11/2013 a 15/1/2014. Para tanto, foi utilizada a técnica de análise de conteúdo para avaliar respostas de questões abertas, conforme indicado por Bardin, Reto, and Pinheiro (1979) e Moraes (1999).

(D) Entrevista semiestruturada com o Comandante do $8^{\circ}$ BPMMG, realizada no 
dia 28/1/2014, às 16 horas, na sede do 8o Batalhão, em Lavras - MG, conforme roteiro previamente estabelecido.

(E) Pesquisa na mídia local sobre inovações obtidas durante os anos de 2014 e de 2015 que retratassem os resultados obtidos a partir da utilização da plataforma de ideias pela instituição proponente do desafio.

O objetivo da entrevista foi verificar a motivação do proponente para a utilização da inovação aberta e sua percepção em relação aos resultados das análises quantitativas e de conteúdo das ideias, realizadas nas etapas (B) e (C) da pesquisa. As informações obtidas por meio da entrevista foram transcritas e enviadas ao entrevistado para validação.

Para realizar a análise de conteúdo, citada na etapa (C), foi estabelecido que cada ideia representaria uma unidade de análise; depois as ideias foram agrupadas, conforme suas similaridades, o que permitiu a formação de categorias. Amostra de $15 \%$ das ideias passou por três iterações de validação, conforme a triangulação de investigador de Denzin (1970), na qual mais de um pesquisador revisa autonomamente uma amostra verificando a exatidão da categorização.

Após a terceira iteração, foi obtido um erro de $4 \%$ das categorizações, e a categorização foi considerada válida. Depois desses testes e do refinamento das categorias, chegou-se à Tabela 1 de categorização, composta por nove grupos temáticos, divididos em outros três grupos, formando três categorias macro (comunidades, polícia e outras instituições). Os comentários também foram agrupados em categorias, seguindo o mesmo procedimento. 
DESAFIO DE IDEIAS PARA O GOVERNO ABERTO: O CASO DA POLÍCIA MILITAR DE MINAS GERAIS - BRASIL

Tabela 1. Descrição das categorias utilizadas na etapa de categorização das ideias

\begin{tabular}{|c|c|c|c|}
\hline \multicolumn{2}{|c|}{$\begin{array}{l}\text { Categorias e } \\
\text { subcategorias }\end{array}$} & Descrição da subcategoria & Exemplos de unidade de análise \\
\hline \multirow{3}{*}{ 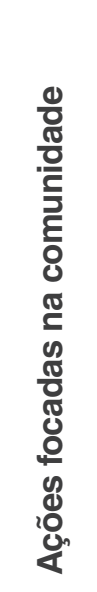 } & Organização (C1) & $\begin{array}{l}\text { Ideias sobre maneiras pelas quais as } \\
\text { comunidades são estimuladas a se auto- } \\
\text {-organizarem. }\end{array}$ & $\begin{array}{l}\text { "[...] os vizinhos, ao verificarem a presen- } \\
\text { ça de suspeitos nas imediações, passa- } \\
\text { rem a fazer sinais de apito como senha } \\
\text { para os demais vizinhos." }\end{array}$ \\
\hline & $\begin{array}{l}\text { Canais de comunicação } \\
\text { e conscientização (C2) }\end{array}$ & $\begin{array}{l}\text { Ideias sobre mecanismos de comunica- } \\
\text { ção, troca de conhecimentos e conscien- } \\
\text { tização da comunidade. }\end{array}$ & $\begin{array}{l}\text { "Verificar a criação de Conseps [Conse- } \\
\text { Iho Comunitário de Segurança Pública] } \\
\text { por vários bairros." }\end{array}$ \\
\hline & $\begin{array}{l}\text { Comportamento } \\
\text { (C3) }\end{array}$ & $\begin{array}{l}\text { Ideias sobre como cada cidadão deve se } \\
\text { comportar para estar protegido. }\end{array}$ & $\begin{array}{l}\text { "Evitar usar maletas de notebook. O me- } \\
\text { Ihor é usar uma bolsa normal que não } \\
\text { aparente estar com um aparelho eletrô- } \\
\text { nico." }\end{array}$ \\
\hline \multirow{3}{*}{ 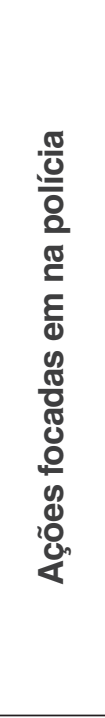 } & $\begin{array}{l}\text { Logística e prestação de } \\
\text { serviços (P1) }\end{array}$ & $\begin{array}{l}\text { Ideias sobre melhorias ou realocação de } \\
\text { recursos da polícia e melhor prestação } \\
\text { de serviços. }\end{array}$ & $\begin{array}{l}\text { "Colocar mais guaritas e aumentar o nu- } \\
\text { mero de veículos de ronda da polícia [...]" }\end{array}$ \\
\hline & $\begin{array}{l}\text { Recursos humanos } \\
\text { (P2) }\end{array}$ & $\begin{array}{l}\text { Ideias sobre investimentos e valorização } \\
\text { de recursos humanos pela instituição. Não } \\
\text { pertence a essa categoria a alocação de } \\
\text { recursos humanos (logística), mas, sim, } \\
\text { sua capacitação e valorização. }\end{array}$ & $\begin{array}{l}\text { "[...] quando [os policiais] são bem remu- } \\
\text { nerados, com cursos motivadores, acom- } \\
\text { panhamento médico e psicológico poderá } \\
\text { (sic) exercer as suas atividades com mais } \\
\text { concentração e dedicação." }\end{array}$ \\
\hline & $\begin{array}{l}\text { Gestão da inovação } \\
\text { (P3) }\end{array}$ & $\begin{array}{l}\text { Ideias sobre a adoção de novas tecnolo- } \\
\text { gias de informação ou por pesquisas que } \\
\text { visem à inovação nos serviços prestados. }\end{array}$ & $\begin{array}{l}\text { “[...] criação de um aplicativo que } \\
\text { avise no mesmo instante a ocorrência de } \\
\text { um assalto [e] a posição geográfica e en- } \\
\text { viando a informação direto para todas as } \\
\text { viaturas que estão no patrulhamento. [...]" }\end{array}$ \\
\hline \multirow{3}{*}{ 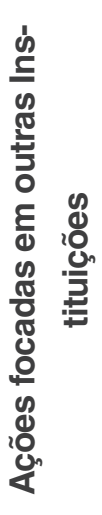 } & Legislação (01) & $\begin{array}{l}\text { Ideias que dependem da criação de leis } \\
\text { ou órgãos públicos, bem como da melhor } \\
\text { aplicação dessas leis. }\end{array}$ & $\begin{array}{l}\text { "[...] poderia ser criada uma lei nacional } \\
\text { que proibisse a fabricação e comercializa- } \\
\text { ção de armas de brinquedo." }\end{array}$ \\
\hline & $\begin{array}{l}\text { Espaços públicos } \\
(\mathrm{O} 2)\end{array}$ & $\begin{array}{l}\text { Ideias sobre a manutenção ou criação de } \\
\text { espaços físicos públicos. }\end{array}$ & $\begin{array}{l}\text { "Criação de um centro de apoio para } \\
\text { aconselhamento [...] e prevenção/reabili- } \\
\text { tação para (potenciais) criminosos." }\end{array}$ \\
\hline & $\begin{array}{l}\text { Parcerias } \\
\text { (O3) }\end{array}$ & $\begin{array}{l}\text { Ideias sobre parcerias entre órgãos pú- } \\
\text { blicos e privados para prestar serviços à } \\
\text { comunidade. }\end{array}$ & $\begin{array}{l}\text { "Firmar parcerias com os grandes super- } \\
\text { mercados, para que, quando eles dis- } \\
\text { tribuírem seus panfletos de promoções, } \\
\text { distribuam também dicas do 8 BPMMG." }\end{array}$ \\
\hline
\end{tabular}

Fonte: Elaborada pelos autores. 
O resultado da classificação das ideias foi apresentado na Figura 2, por meio das porcentagens de ideias enviadas por policiais e cidadãos em cada categoria. A porcentagem foi calculada separadamente para cada perfil, ou seja, do total de ideias enviadas por policiais, foi calculada qual porcentagem se relacionava a cada categoria. Posteriormente, o mesmo foi realizado para as ideias enviadas por cidadãos.

Os demais resultados desta pesquisa foram apresentados por meio da transcrição de trechos da entrevista com o Comandante da instituição proponente do desafio e da pesquisa em mídias locais sobre os impactos da utilização da plataforma de envio de ideias.

\section{RESULTADOS}

Nesta seção, as questões de pesquisa foram respondidas: "Como o 8 BPMMG, conhecido amplamente por sua estrutura formal e processos rígidos, fez uso de plataforma de inovação aberta para fortalecer a colaboração, pilar do governo aberto?" E, ainda, "Quais os resultados da aplicação dos conhecimentos absorvidos nessas iniciativas?".

Para isso, inicialmente, foi apresentado como o 8 BPMMG utilizou a plataforma Prêmioldeia em prol da inovação e, na sequência, foram apresentados os benefícios dessa iniciativa, que foram: a colaboração entre agentes da polícia e cidadãos, fortalecendo o pilar da colaboração em um gover- no aberto; e como a percepção dos cidadãos em relação aos serviços, programas e problemas enfrentados pela PMMG foi absorvida e utilizada pela instituição.

Prêmioldeia - Uma Plataforma para Envio de Ideias

O Prêmioldeia é um aplicativo de software para gestão de ideias, apoiado nos conceitos de inovação aberta, gamificação e redes sociais (Prêmioldeia, 2015). A inovação aberta é identificada pela utilização da técnica de crowdsourcing, por meio da qual diferentes atores são estimulados a enviar ideias de soluções para um problema comum a eles. A gamificação adota a estrutura de um jogo no qual uma instituição, do Estado ou não, expõe um problema social para que a comunidade on-line envie ideias que visem a sua resolução. Em troca, a instituição proponente do desafio oferece uma premiação para a melhor ideia.

As redes sociais, como Facebook, LinkedIn, Twitter, Instagram e Google+, são utilizadas para direcionar o aplicativo para indivíduos previamente conectados e que tenham relação com o problema proposto.

A Figura 1 mostra uma tela do Prêmioldeia, que, quando acessada por um usuário, the permite visualizar o nome do desafio, o tempo em que ele estará disponível para participação e as ideias enviadas por outros usuários. 
Figura 1 Tela da plataforma de gestão de ideias

Idéias e estratégias para incrementar a ação da PM em redes de proteção ou criação de novas redes

Este desafio termina em 101d $14 \mathrm{~h} 57 \mathrm{~m} 26 \mathrm{~s}$

\section{: Mais detalhes Enviar Ideia}

Rede restrita a bairros, comunidades ou grupos de compartilhamento de fotos -

$26 / 02 / 2014$ às $20: 00: 58$

Identificação do autor da ideia

Pessoas de dentro da mesma rede social (grupo do bairro, repúblicas, igreja..) e entidades policiais podem postar fotos na rede no momento que algo de anormal ou estranho acontece, como por exemplo veículo parado a vários dias em determinado local, pessoas desconhecidas frequentando lugares comuns etc. As entidades policiais, também presentes na rede, podem identificar suspeitos ou atividades suspeitas baseadas nestas fotos e posteriormente iniciam a averiguação.

Fonte: Prêmioldeia (2015).

Conforme mostra a Figura 1, os usuários escolhem de qual desafio vão participar. As ideias ficam expostas para que outros participantes opinem ou sugiram melhorias por meio da funcionalidade "Comentários". Os outros participantes também podem demonstrar sua concordância ou discordância com a ideia, por meio das opções "Curti" e "Não curti". Em suma, há três maneiras de participação: enviando ideias, comentando as ideias dos outros participantes e avaliando essas ideias por meio das opções "Curti" e "Não curti".

No Prêmioldeia, um desafio de ideias tem, em média, dois meses de duração, nos quais há uma tendência de aumento progressivo do número de participantes, graças à estrutura de rede do Facebook que permite que cada participação de um usuário seja divulgada em sua rede de contatos. Terminado o prazo do desafio, o participante que mais colaborou com o desafio é premiado pela instituição proponente. A escolha do ganhador é realizada de maneira indireta pelos demais participantes do jogo. Os participantes vão acumulando pontos de acordo com suas ações no aplicativo e com a colaboração e aprovação de suas ideias por outros participantes do desafio.

Oitavo Batalhão da Polícia Militar de Minas Gerais ( $8^{\circ} \mathrm{BPMMG}$ ) e o Desafio Cidade Segura

A Polícia Militar de Minas Gerais é a instituição responsável por executar as políticas em segurança pública no estado de Minas Gerais (Brasil, 2013) e é detentora do uso legal da força para a garantia do poder de polícia; possui estrutura hierárquica, processos formais rígidos e marcados fortemente pela cultura militar (GERAIS, 2012). O $8^{\circ}$ BPMMG é um dos 58 Batalhões Operacionais responsáveis pelo policiamento ostensivo geral em Minas Gerais. É uma instituição militar tradicional, localizada na cidade de Lavras-MG, e atende a 405.820 cidadãos distribuídos em 25 cidades da região (Santos et al., 2015). Assim como outras insti- 
tuições militares, o batalhão é conhecido amplamente por sua estrutura hierárquica, processos formais rígidos e marcados fortemente pela cultura militar, o que tende a distanciá-la dos cidadãos atendidos por ela.

Apesar da rigidez e formalidade de sua estrutura, os artigos que se referem especificamente ao 8 BPMMG apresentam iniciativas que demonstram 0 interesse desse órgão na modernização de seus processos (Santos, Zambalde, \& Brito, 2006), no intercâmbio de conhecimentos entre seus membros internos (Pereira, Santos, \& Brito, 2010), no gerenciamento de indicadores estratégicos em segurança pública (Pereira et al., 2010) e, mais recentemente, na busca por uma comunicação bidirecional com os cidadão atendidos por ele (Santos et al., 2015).

Segundo Santos, em prol da inovação e democratização, a instituição criou em 23/9/2013, na plataforma Prêmioldeia, uma comunidade on-line denominada "Comunidade Cidade Segura", na qual se inscreveram, até 20/11/2013, 2.515 usuários. A essa comunidade, foram propostos desafios de envios de ideias para resolução de problemas do setor público, entre eles, o objeto deste estudo: o desafio de ideias "Estratégias e ações para reduzir os crimes contra o patrimônio".

O desafio funcionou da seguinte maneira: a instituição proponente - 8을 BMG - lançou uma questão on-line: "Como reduzir os crimes contra o patrimônio?", por meio da qual, segundo Santos2, esperava-se atender a duas demandas principais, que vão ao encontro das necessidades reconhecidas pela sociedade. A primeira demanda é a necessidade de democratização do planeja- mento estratégico da instituição, adquirindo conhecimentos da sociedade: "Não somos donos da verdade, e a percepção de quem vive diretamente os problemas pode auxiliar a buscar novas soluções".

A segunda demanda refere-se especificamente a combater um problema compartiIhado por toda a sociedade, que é a falta de segurança pública, apontada em pesquisa nacional realizada em junho de 2013 como o maior problema social do Brasil na atualidade (Ibope, 2014). E, segundo constatado na entrevista, é o tipo de crime que mais incomoda os cidadãos, por ser de resolução complexa e vir crescendo em função do aumento do uso de drogas.

Exposto o problema, os usuários da Comunidade Cidade Segura enviaram e avaliaram ideias e, em troca, receberam pontos a cada participação na plataforma. Cada uma dessas interações contabilizou um número de pontos para o participante, e, ao final do desafio, o participante que mais acumulou pontos recebeu um prêmio da instituição.

O referido desafio de ideias, durante os dois meses em que esteve disponível, contou com 11.000 acessos, 2.515 usuários cadastrados, 883 usuários com participações na plataforma, entre cidadãos e policiais, enviando 336 ideias, 1.243 comentários e 19.738 avaliações das ideias. Os prêmios oferecidos pelo proponente foram dois aparelhos de televisão, entregues ao primeiro e ao segundo lugar.

O Fortalecimento da Colaboração, Pilar do Governo Aberto

O estímulo à colaboração foi considerado 
positivo pela instituição e pôde ser identificado em dois aspectos: por meio do ganho em termos de conhecimento a respeito da percepção dos cidadãos e membros da própria polícia em relação ao tema; e, ainda, pela oportunidade de interação e troca de conhecimentos entre cidadãos e policiais, gerando soluções a serem implantadas pela instituição.

Por meio das discussões espontâneas, oportunizadas pelo desafio de ideias, conhecimentos considerados de grande valia para o planejamento estratégico da instituição foram extraídos (Santos et al., 2015). A análise de conteúdo das ideias postadas permitiu extrair a percepção dos participantes sobre quem são os atores responsáveis pelo combate ao crime contra o patrimônio e sobre quais ações eles devem realizar para resolver o problema, conforme disposto na Figura 2.

Para detalhar a análise, os participantes do desafio foram divididos em policiais e cidadãos. Essa divisão foi realizada porque, segundo a direção da instituição, um dos ganhos do desafio foi a captação da visão tanto do público atendido pelo batalhão quanto dos atores responsáveis pela execução das ações desenvolvidas pela instituição.

Figura 2. Ideias divididas em categorias

\section{Porcentagem de ideias divididas em categorias}

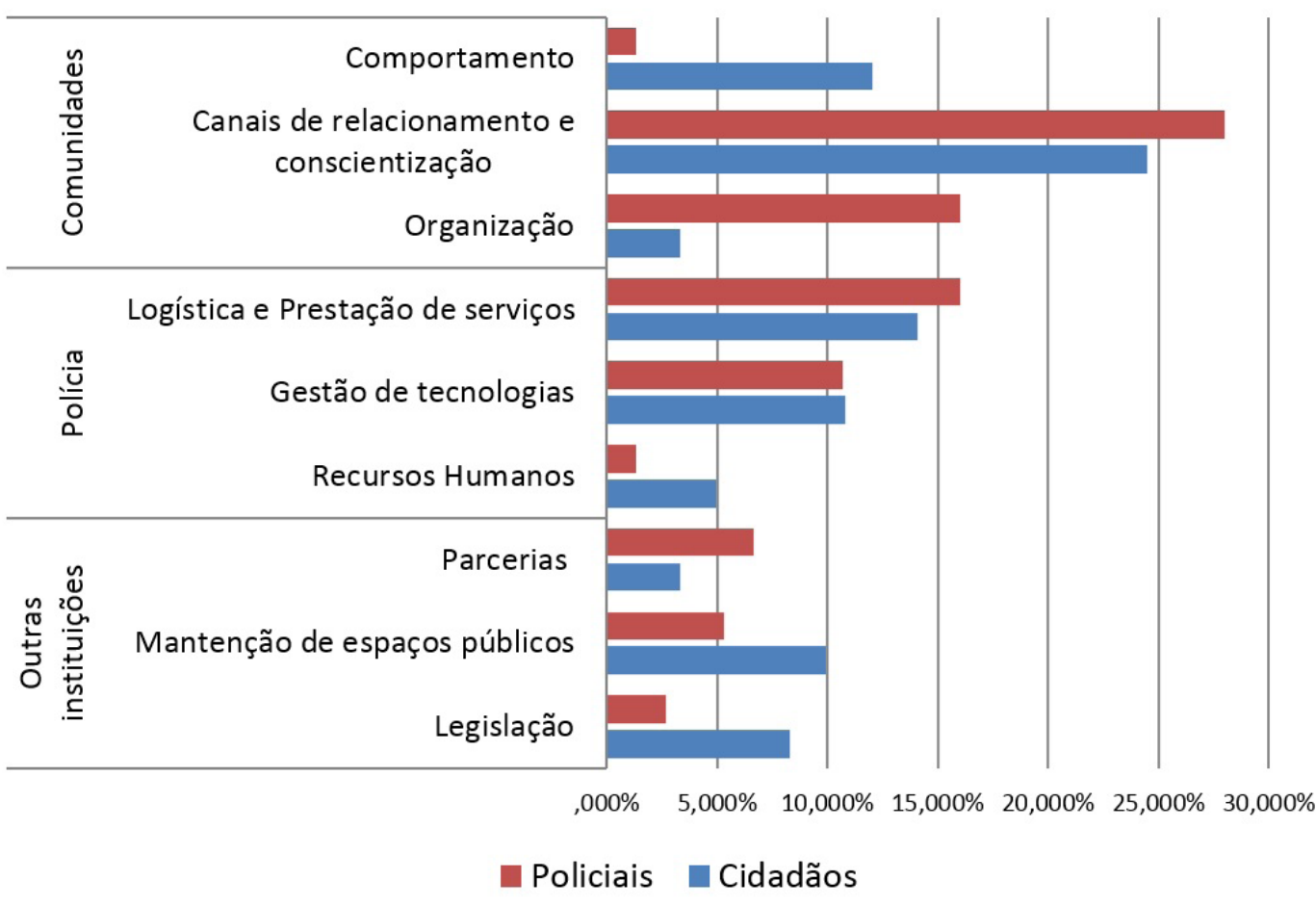

Fonte: Dados da pesquisa. 
$\mathrm{Na}$ visão geral dos participantes, os canais de relacionamento e conscientização seriam a principal estratégia para combater crimes contra o patrimônio, incluindo palestras, debates, campanhas, projetos sociais e ações nas redes sociais que levassem a própria comunidade, em parceria com a Polícia Militar, a criar ações em prol da segurança pública. $\mathrm{Na}$ opinião dos cidadãos, também se destacaram ideias relacionadas ao comportamento individual de cada cidadão, evitando situações perigosas; melhorias na prestação de serviços; e, ainda, ideias sobre gestão de tecnologias, softwares de geoprocessamento nas viaturas, criação de aplicativos para celular, carros-isca com GPS.

As ideias propostas pelos policiais ficaram bem distribuídas em duas categorias: logística e prestação de serviços, e organização da comunidade. E ambos os grupos concordam que é necessária a criação de mecanismos que proporcionem maior interação entre eles. Essa percepção, aliada ao grande número de ideias referentes a criação de redes de participação, legitimou uma inovação da instituição proponente apresentada na seção seguinte, e fortalece a ideia de que os grupos se interessam em colaborar.

Segundo apresentado na entrevista, mais do que captar ideias de inovação, o grande ganho sob o ponto de vista da colaboração foi a oportunidade de interação entre os agentes públicos da instituição e os cidadãos, receptores de seus serviços. Do total de ideias, 76,95\% foram postadas por cidadãos e $20,05 \%$, por policiais e, em média, cada policial contribuiu com 2,33 ideias e cada cidadão contribuiu com 4,85 ideias.

A troca de conhecimentos entre os agentes de polícia e os demais cidadãos foi importante para que as ideias que descreviam projetos já existentes não fossem consideradas inovadoras e, em vez disso, os autores dessas ideias se questionassem sobre as limitações das políticas públicas já existentes e sobre como enfrentar tais limitações para a melhoria e divulgação dessas políticas públicas.

Um exemplo de ideia, inicialmente não considerada inovadora e que foi discutida pelos participantes, foi: "Criar associações de bairro," postada por um cidadão e não considerada inovadora pelos policiais, que argumentaram que tais associações já existem. Porém, a ideia impulsionou a discussão sobre as limitações das associações, como a baixa participação da sociedade, abordada por um policial: "[...] a grande dificuldade [...] é a falta de participação da maioria dos moradores de uma comunidade." Nesse sentido, foram discutidos os possíveis motivos para a não participação, como a "falta de divulgação sobre essas associações", levantada por um cidadão, e "a falta de recursos humanos para alavancá-las", argumento utilizado por um policial (fragmentos de comentários postados na plataforma). O resultado da discussão foi uma ideia de inovação de criação de novos mecanismos de interação, voltados para públicos específicos. Nesse sentido, foi criado um projeto denominado "Redes de repúblicas protegidas", apresentado na sequência, e, atualmente, as ações das associações de bairro são divulgadas em estações de rádio locais. Enquanto uma pesquisa on-line unidirecional poderia retornar a ideia inicial e não atrativa de "Criar associações de bairro", o fluxo bidirecional de informações permitiu a melhoria na divulgação de projetos existentes e a construção de uma nova ideia, com- 


\section{DESAFIO DE IDEIAS PARA 0 GOVERNO ABERTO: 0 CASO DA POLÍCIA MILITAR DE MINAS GERAIS - BRASIL}

posta pela visão dos agentes públicos e da sociedade.

Ao passo que os participantes enviaram ideias para a resolução dos problemas, outros cidadãos participaram no crivo dessas ideias, avaliando-as como positivas ou negativas e demonstrando quais ideias são mais bem recebidas pela amostra da sociedade que participa do desafio. Nesse caso, o fluxo de informações recebidas foi no sentido mais de participação do que de colaboração, pois o sistema de avaliação se assemelha a uma consulta pública on-line de questões objetivas, na qual questões ou ideias são postas à apreciação do público e há retorno de respostas padronizadas. Apesar de não contribuir para a colaboração, a avaliação das ideias permitiu verificar quais delas foram mais populares e, segundo afirmado na entrevista, tais ideias receberam destaque nas discussões sobre o que poderia ser implementado.

A partir das 19.871 avaliações, entre as ideias que mais se destacaram, estão as que tratam sobre "Gestão de tecnologias" e, mais especificamente, aquelas associadas à colocação de câmeras foram as que mais se repetiram. Na categoria "Canais de comunicação e conscientização", as ideias propondo a criação de novas redes comunitárias foram as mais bem avaliadas. Esse destaque atribuído pela sociedade impulsionou duas inovações no 8 BPMMG, logo após o desafio, conforme apresentado na seção seguinte.

Resultados Gerados e Retorno aos Participantes

Dois anos após o encerramento do desafio de ideias, as pesquisas na mídia local e na plataforma Prêmioldeia permitem um estudo em médio prazo dos resultados do desafio e de qual foi o retorno dado aos participantes. Durante o desafio de ideias, foram propostas 336 ideias e, entre elas, algumas levaram à criação de projetos que foram divulgados aos participantes dos desafios, bem como à comunidade beneficiária de tais projetos.

Um dos temas mais recorrentes nas ideias foi a maior interação entre polícia e comunidade, 20 das 336 ideias tratavam da formação de redes de relacionamento entre poder público e sociedade. As ideias geradas no desafio criaram uma nova política pública: 0 programa "Redes de repúblicas protegidas" ${ }^{1}$ (Santos et al., 2015). Esse projeto segue a mesma filosofia da "Rede de vizinhos protegidos", citada como modelo em seis ideias apresentadas no desafio, porém voltada para os estudantes, público muito significativo na área de atuação do batalhão. Dois anos depois do desafio, o programa conta com a participação de 450 repúblicas estudantis que se reúnem periodicamente com agentes de segurança pública, reforçando a interação, e atuam juntos no combate ao furto e violação das moradias estudantis.

Outro tema recorrente foi a utilização de câmeras para coibir crimes contra o patrimônio; 20 ideias citavam as câmeras em locais estratégicos, câmeras falsas, nos bairros, em veículos, entre outras. Em 6 de fevereiro de 2014, dois meses depois de finalizado o desafio, foi iniciada a recolocação de câmeras em 12 pontos da cidade (Brasil, 2014). E, em 23 de março de 2014, foi divulgado pela imprensa local o resultado positivo da utilização das câmeras no combate a crimes (Zanforlin, 2014). Nesse caso, as ideias 
se mostraram pertinentes por envolverem projetos que obtiveram resultados positivos coibindo os crimes contra o patrimônio.

Entre as ideias relacionadas à gestão da inovação, dois participantes citaram a utilização de veículos aéreos não tripulados (drones) com câmeras e a criação de uma central de monitoramento. "No dia seguinte, quando o Comandante do 8을 Batalhão viu a ideia, imediatamente escreveu um projeto para o prefeito da cidade, que consentiu na ideia e apresentou o projeto para aprovação da Câmara Municipal" (Santos et al., 2015). A utilização dos drones foi destaque na mídia regional como uma inovação na apreensão de plantações de maconha na região². Dessa forma, foi uma inovação que impactou a resolução do principal problema apontado pela instituição pública que propôs o desafio: a criminalidade devido ao tráfico de drogas.

A utilização do desafio de ideias, também, gerou para a instituição proponente uma postura mais inclusiva, a partir da implementação de algumas ideias e da criação de novos desafios para explorar as melhores ideias obtidas nessa primeira experiência. Um exemplo foi a criação do desafio "Ideias e estratégias para incrementar a ação da PM em redes de proteção ou criação de novas redes", criado a partir do destaque atribuído ao tema pelos participantes do desafio estudado neste artigo.

Também foi criado um desafio para apoiar um projeto que já estava sendo desenvolvido pela instituição: a prevenção de acidentes de trânsito. Segundo apresentado na entrevista, o desafio "Ações e estratégias para a Lei Seca" reforçou a ideia de auto-or- ganização da sociedade e resultou em diversas ideias sobre como a própria comunidade poderia agir para evitar que pessoas dirijam alcoolizadas. Assim, a criação de novos desafios de ideias sinaliza para a possibilidade de difusão dessas práticas participativas e o prosseguimento da instituição na busca de práticas de gestão mais democráticas.

A utilização da plataforma de envio de ideias por uma instituição militar gerou dois resultados principais no que se refere ao atendimento das demandas que o motivaram. Em primeiro lugar, houve uma inovação na maneira de captar a informações dos cidadãos em relação aos serviços, programas e problemas enfrentados pelo 8 BPMMG e na maneira de possibilitar a interação entre agentes da polícia e cidadãos. E o outro resultado positivo foi a implementação de várias ideias, principais produtos gerados nesse processo, que resultaram em ações ou em projetos que colaborem para o atendimento da necessidade social em questão.

\section{Limitações da Utilização da Plataforma para} o Estímulo à Colaboração

Após a análise dos resultados da utilização da plataforma de ideias em médio prazo, foram constatadas algumas limitações em sua utilização para promover a colaboração e inovação em instituições públicas. As principais limitações relativas a esse caso foram os efeitos da gameficação, característica inerente a esse tipo de plataforma; além da dificuldade de se alcançarem todos os grupos de cidadãos atendidos.

A análise das ações dos participantes durante o desafio permitiu inferir que, mais do que contribuir para gerar ou aprimorar ideias 
para o bem público, muitos participantes tinham por objetivo pontuar mais no desafio ou auxiliar outros participantes a pontuar, o que é um efeito da gamificação. Por meio da análise do conteúdo dos comentários postados nas ideias, percebe-se que $88,19 \%$ deles não acrescentam novas informações às ideias, são apenas elogios breves sobre a ideia ou comentários dos próprios autores da ideia agradecendo o apoio. Considerando que cada usuário pontua quando recebe comentários em suas ideias, a principal relevância dos comentários curtos é auxiliar determinado participante no jogo.

Assim, apesar da constatação positiva de que a gamificação pode impulsionar a participação dos cidadãos, percebe-se que tais participações podem ser consideradas superficiais, o que também é notado em pesquisas sobre a participação on-line, observação que, inclusive, deu origem à estatística proposta por Nilsen (2006) de que cerca de $90 \%$ dos usuários de plataformas on-line atuam somente como observadores, cerca de $9 \%$ contribuem, mas não tão frequentemente, e somente $1 \%$ tende a contribuir efetiva e rotineiramente para tais plataformas.

Outra limitação é a dificuldade de obter a participação de todos os grupos de cidadãos interessados no tema. Considerando o número total de cidadãos atendidos pela instituição, a participação na plataforma pode ser considerada relativamente baixa; porém, pesquisas anteriores já demonstraram que ainda há dificuldades no acesso à internet por vários grupos da sociedade (Al-Hujran et al., 2015; Conradie \& Choenni, 2014; Neves, 2013), além das típicas dificuldades de se obter a participação popular, oriundas do processo histórico e de formação cultural do povo brasileiro, bem como das políticas fechadas e corporativistas que perduraram no Brasil (Maciel, 2014).

Além dessas limitações, ressalta-se que a utilização da plataforma apresentou resultados positivos na visão da instituição implementadora, mas sua aplicação para a abertura de outras instituições depende de vários fatores, como a sensibilização de outros gestores públicos e a existência de recursos humanos, financeiros e tecnológicos para a utilização dessas plataformas em outros contextos.

\section{DISCUSSÃO E CONCLUSÃO}

Ao longo deste artigo, foram discutidos os ganhos em termos de colaboração e inovação obtidos pelo $8^{\circ}$ Batalhão da Polícia Militar por meio da utilização da plataforma de envio de ideias Prêmioldeia. É perceptível, a partir da análise desses ganhos, que a maior inovação ocorrida foi a nova modalidade de interação que se estabeleceu entre agentes públicos e cidadãos. Essa interação foi importante para que surgisse uma comunicação bidirecional entre os agentes públicos e os cidadãos, construindo juntos soluções para um problema público.

No artigo, conceitos recentes foram explorados, sobretudo os conceitos de inovação aberta e governo aberto. Foi proposto estudar o desafio de ideias como uma inovação, visando fomentar os estudos em inovação aberta também com casos aplicados ao setor público, cujo objetivo não seja o lucro. E foi constatado que a principal inovação obtida foi a própria abertura de uma instituição como a Polícia Militar, conhecida pela rigi- 
dez de suas normas e pela unilateralidade de suas ações.

A abertura da instituição para ouvir os cidadãos atendidos por ela e viabilizar o diálogo entre eles e seus agentes gerou um ambiente colaborativo on-line, que ultrapassa as principais definições de governo eletrônico, não somente por constituir a disponibilização de serviços governamentais on-line, mas também por viabilizar a abertura de uma instituição governamental para a colaboração entre os atores na construção de soluções para problemas públicos.

Artigos mais recentes apontaram a falta de uma colaboração efetiva e de feedback dos governos aos cidadãos como principais problemas na implementação do governo aberto, além da dificuldade de se obter participação e colaboração dos participantes. Apesar das limitações da plataforma, demonstradas na seção anterior, foram considerados ganhos em colaboração e inovação:

- O feedback da instituição por meio da implementação das melhores ideias propostas por cidadãos e da divulgação em mídia local do resultado dessas ideias.

- A atitude de, ao divulgar os resultados positivos de projetos inovadores da instituição, chamar atenção para o fato de que a ideia geradora da inovação partiu da comunidade por meio da plataforma de ideias.

- A interação entre agentes públicos e cidadãos durante o desafio para aprimoramento das propostas, tornando-as o mais viáveis possível.

- A utilização da plataforma para divulgação das reuniões e projetos da instituição no intuído de manter o canal de comuni- cação entre os agentes públicos e cidadãos.

Considerando que o estudo sobre mecanismos que levem à colaboração para o governo aberto ainda é incipiente no Brasil, espera-se que o relato desse caso de inovação motive novas aplicações das metodologias de inovação aberta com finalidades públicas e o crescimento da colaboração entre as instituições públicas e a sociedade na construção de novas políticas públicas.

Como limitações da pesquisa, tem-se que o estudo se restringiu ao $8^{\circ}$ BPMMG, e não é possível que sua conclusão seja generalizada, até pelo fato de que a implementação de iniciativas como essa depende de vários fatores inerentes à instituição.

Trabalhos futuros podem explorar outras aplicações da inovação aberta ao serviço público e compará-las com esta pesquisa, de modo a identificar as limitações e potencialidades dessa estratégia de abertura das instituições públicas.

\section{REFERÊNCIAS}

Abu-Shanab, E. A. (2015). Reengineering the open government concept: An empirical support for a proposed model. Government Information Quarterly, 32(4), 453-463. doi:10.1016/j.giq.2015.07.002

Agner, L. C. (2015). O movimento dos e-governos do Brasil e do Canadá em direção a uma cultura de interfaces centradas no cidadão. Interfaces Brasil/Canadá, 8(1-2), 281294.

Al-Hujran, O., Al-Debei, M. M., Chatfield, A., 


\section{DESAFIO DE IDEIAS PARA 0 GOVERNO ABERTO: 0 CASO DA POLÍCIA MILITAR DE MINAS GERAIS - BRASIL}

\& Migdadi, M. (2015). The imperative of influencing citizen attitude toward e-government adoption and use. Computers in $\mathrm{Hu}$ man Behavior, 53, 189-203. doi:10.1016/j. chb.2015.06.025

Alawneh, A., Al-Refai, H., \& Batiha, K. (2013). Measuring user satisfaction from e-government services: Lessons from Jordan. Government Information Quarterly, 30(3), 277288. doi:10.1016/j.giq.2013.03.001

Bardin, L., Reto, L. A., \& Pinheiro, A. (1979). Análise de conteúdo. Lisboa, Portugal: Edições 70.

Bloch, C., \& Bugge, M. M. (2013). Public sector innovation: From theory to measurement. Structural Change and Economic Dynamics, 27, 133-145. doi:10.1016/j.strueco.2013.06.008

Borman, M., \& Janssen, M. (2013). Reconciling two approaches to critical success factors: The case of shared services in the public sector. International Journal of Information Management, 33(2), 390-400. doi: 10.1016/j.jijnfomgt.2012.05.012

Cavalheiro, G. M. d. C., \& Joia, L. A. (2014). Towards a heuristic frame for transferring e-government technology. Government Information Quarterly, 31(1), 195-207. doi: http:// dx.doi.org/10.1016/j.giq.2013.09.005

Chesbrough, H.W. (2003). Open innovation: The new imperative for creating and profiting from technology. Boston, EUA: Harvard Business Press.

Conradie, P., \& Choenni, S. (2014). On the barriers for local government releasing open data. Government Information Quarterly, 31, S10-S17.

Constant, V. S. (2009). Projetos e relatórios de pesquisa em administração. São Paulo, SP: Atlas, 2000.

Cordella, A., \& lannacci, F. (2010). Information systems in the public sector: The e-government enactment framework. The Journal of Strategic Information Systems, 19(1), 5266. doi: 10.1016/j.jsis.2010.01.001

Cunha, M. A. (2010). Governo Eletrônico no Brasil: Avanços e impactos na sociedade brasileira. Pesquisa sobre o uso das tecnologias da informação e da comunicação no Brasil 2005-2009, 1-73.

Deng, Z., Lin, Y., Zhao, M., \& Wang, S. (2015). Collaborative planning in the new media age: The Dafo Temple controversy, China. Cities, 45, 41-50. doi: 10.1016/j.cities.2015.02.006

Denzin, N. K. (1970). The research act: $A$ theoretical introduction to sociological methods: Transaction publishers. New Jersey. United States of America.

Gonzalez, R., Llopis, J., \& Gasco, J. (2013). Innovation in public services: The case of Spanish local government. Journal of $\mathrm{Bu}$ siness Research, 66(10), 2024-2033. doi: 10.1016/j.jbusres.2013.02.028

Hernández, G. I., Juan Fuente, A. A., Labra-Gayo, J. E., Pérez, B. L., \& Núñez-Valdez, E. R. (2015). Knowledge-based public service transactions: An intelligent model-driven approach in co-learning contexts. Computers in Human Behavior, 51, Part B, 1032-1041. doi: 10.1016/j.chb.2014.09.044 
Hopkins, R. (2011). What is Crowdsourcing? In S. Paul (Ed.), A guide to open innovation and crowdsourcing (Vol. 1, 5-14). London, UK: KoganPage.

Instituto Brasileiro de Opinião Pública e Estatística. (2013). 89\% dos manifestantes não se sentem representados por partidos. Disponível em: <http://www.ibope.com.br/pt-br/ noticias/Paginas/89-dos-manifestantes-nao-se-sentem-representados-por-partidos. aspx> Acesso em: 12.dez.2016.

Instituto Brasileiro de Opinião Pública e Estatística. (2014). Pesquisa de opinião pública sobre as manifestações. Disponível em: <http://www.ibope.com.br/pt-br/noticias/ Paginas/75-dos-brasileiros-sao-favoraveis-asanifestaco-publicas.aspx> Acesso em: 12.dez.2016.

Karakiza, M. (2015). The Impact of Social Media in the Public Sector. Procedia - Social and Behavioral Sciences, 175, 384-392. doi: 10.1016/j.sbspro.2015.01.1214

Karunasena, K., \& Deng, H. (2012). Critical factors for evaluating the public value of e-government in Sri Lanka. Government Information Quarterly, 29(1), 76-84. doi: 10.1016/j. giq.2011.04.005

Lee, G., \& Kwak, Y. H. (2012). An Open Government Maturity Model for social media-based public engagement. Government Information Quarterly, 29(4), 492-503.

Linders, D. (2012). From e-government to we-government: Defining a typology for citizen coproduction in the age of social media. Government Information Quarterly, 29(4),
446-454. doi: 10.1016/j.giq.2012.06.003

Martins, T. C. M., \& Bermejo, P. H. D. S. (2015). Inovação aberta para a coprodução de inovações no setor público. (Dissertação de mestrado - Universidade Federal de Lavras) Meijer, A., \& Bekkers, V. (2015). A metatheory of e-government: Creating some order in a fragmented research field. Government Information Quarterly, 32(3), 237-245. doi: 10.1016/j.giq.2015.04.006

Meijer, A. J., Curtin, D., \& Hillebrandt, M. (2012). Open government: Connecting vision and voice. International Review of Administrative Sciences, 78(1), 10-29.

Moraes, R. (1999). Análise de conteúdo. Educação, 22(37), 7-32.

Neves, O. M. C. (2013). Evolução das políticas de governo aberto no Brasil. In: congresso consad de gestão pública, 6, 2013, Brasília. Anais

Nielsen, J. (2006). The 90-9-1 rule for participation inequality in social media and online communities. Disponível em: https://www. nngroup. com/articles/participation-inequality/. Último acesso: [last accessed Sept. 16, 2014].

Organisation for Economic Co-operation and Development (2015). Government at a glance 2015. Paris, France: OECD. Recuperado de http://www.oecd.library.org/governance / government-at-a-glance_22214399;jsession id=1a8ovbtpuitwj.x-oecd-live-03.

Osman, I. H., Anouze, A. L., Irani, Z., Al-Ayoubi, B., Lee, H., Balcı, A., ... Weerakkody, V. (2014). COBRA framework to evalua- 
te e-government services: A citizen-centric perspective. Government Information Quarterly, 31(2014), 243-256. doi: 10.1016/j. giq.2013.10.009

Panagiotopoulos, P., Al-Debei, M. M., Fitzgerald, G., \& Elliman, T. (2012). A business model perspective for ICTs in public engagement. Government Information Quarterly, 29(2), 192-202. doi: 10.1016/j.giq.2011.09.011

Parvanta, C., Roth, Y., \& Keller, H. (2013). Crowdsourcing 101: A Few Basics to Make You the Leader of the Pack. Health Promotion Practice, 14(2), 163-167. doi: $10.1177 / 1524839912470654$

Pereira, M. C., Santos, A. C., \& Brito, M. J. (2010). Tecnologia da informação e Gestão do Conhecimento em uma Organização Militar: Uma abordagem interpretativa. GESTÃO. Org-Revista Eletrônica de Gestão Organizacional, 3(3), 166-181.

Pinho, J. D. (2008). Investigando portais de governo eletrônico de estados no Brasil: Muita tecnologia, pouca democracia. Revista de Administração Pública, 42(3), 471493.

Prêmioldeia. (2015). Prêmioldeia. Recuperado de: www.premioideia.com

Rocha, D. G. da, \& Oliveira, G. F. de. (2015). A prestação de contas ao Tribunal de Contas da União no contexto do open government partnership. Revista do TCU, 132, 48-55.

Santos, A. C., Zambalde, A. L., \& Brito, M. J. (2006). Universidade Corporativa: Uma estratégia de educação corporativa da polícia militar. In Anais do Workshop de Informáti- ca na Escola (Vol. 1, No. 1). Disponível em: < http://www.br-ie.org/pub/index.php/wie/issue/view/31> Acesso em: 12.dez.2016.

Santos, A. C., Zambalde, A. L., Veroneze, R. B., Botelho, G. A., \& Bermejo, P. H. D. S. (2015). Open Innovation and Social Participation: A Case Study in Public Security in Brazil. In: international conference on electronic government and the information systems perspective (pp. 163-176). Springer International Publishing. Valence - Spain.

Seltzer, E., \& Mahmoudi, D. (2013). Citizen Participation, Open Innovation, and Crowdsourcing: Challenges and Opportunities for Planning. Journal of Planning Literature, 28(1), 3-18. doi: 10.1177/0885412212469112

Sieber, R. E., \& Johnson, P. A. (2015). Civic open data at a crossroads: Dominant models and current challenges. Government Information Quarterly, 32(3), 308-315. doi: 10.1016/j.giq.2015.05.003

Simms, J. R. (2006). Technical and social innovation determinants of behaviour. Systems Research and Behavioral Science, 23(3), 383-393.

Song, M., \& Guan, Y. (2015). The electronic government performance of environmental protection administrations in Anhui province, China. Technological Forecasting and Social Change, 96, 79-88. doi: 10.1016/j.techfore.2014.10.001

Sun, H., Fang, Y., \& Hsieh, J. J. P.-A. (2014). Consuming information systems: An economic model of user satisfaction. Decision Support Systems, 57, 188-199. doi: 10.1016/j. dss.2013.09.002 
Sun, P.-L., Ku, C.-Y., \& Shih, D.-H. (2015). An implementation framework for E-Government 2.0. Telematics and Informatics, 32(3), 504-520. doi: 10.1016/j.tele.2014.12.003

Vaz, J. C., Ribeiro, M. M., \& Matheus, R. (2010). Dados governamentais abertos e seus impactos sobre os conceitos e práticas de transparência no Brasil. Cadernos PPG-AU/UFBA, 9(1), 45-62.

Veljković, N., Bogdanović-Dinić, S., \& Stoimenov, L. (2014). Benchmarking open government: An open data perspective. Government Information Quarterly, 31(2), 278-290. doi: 10.1016/j.giq.2013.10.011

West, J., Salter, A., Vanhaverbeke, W., \& Chesbrough, H. (2014). Open innovation: The next decade. Research Policy, 43(5), 805-811.

Wijnhoven, F., Ehrenhard, M., \& Kuhn, J. (2015). Open government objectives and participation motivations. Government Information Quarterly, 32(1), 30-42.

Wise, S., Miric, M., \& Gegenhuber, T. (2011). COINs for Government: Collaborative Innovation Networks used in nascent US Government initiatives. Procedia - Social and
Behavioral Sciences, 26(0), 136-146. doi: 10.1016/j.sbspro.2011.10.570

Yin, R. K. (2003). Case Study Research: Design and Methods (vol.5). Sage Publications. Thousand Oaks, CA.

Zanforlin, B. (2014). Homem é flagrado vendendo drogas em Lavras. Recuperado de: http://www.alterosa.com.br/app/ varginha/noticia/jornalismo/alterosa-em-alerta-vg/2014/03/24/noticia-alterosa-em-alerta-vg,109849/homem-e-flagrado-vendendo-drogas-em-lavras.shtml

\section{Notas}

1. Algumas notícias da mídia local: http://g1.globo.com/ mg/sul-de-minas/noticia/2014/02/rede-de-protecao-de-republicas-conta-com-aplicativo-para-celulares. html; https://www.youtube.com/watch?v=znuNwoPSf-c; http://www.ufla.br/ascom/2014/11/20/rede-de-republicas-protegidas-pm-fara-reuniao-com-estudantes-interessados-em-participar/

2. http://g1.globo.com/mg/sul-de-minas/jornal-da-eptv/videos/t/edicoes/v/com-ajuda-de-drone-policia-encontra-plantacao-de-maconha-em-lavras/3904777/ http://www.hojeemdia.com.br/horizontes/pm-de-minas-usara-drones-e-ganhara-reforco-de-450-militares-1.294877 